\title{
Editorial
}

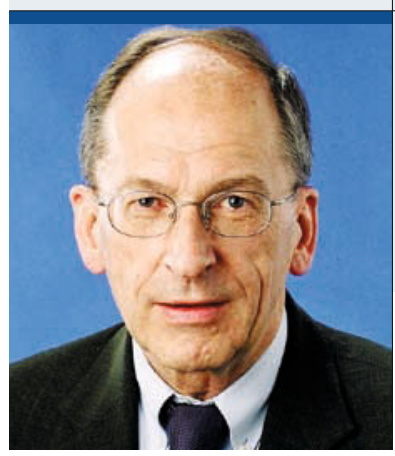

„Die gesetzlichen und die Honorar-vorgaben belohnen den

Aktionismus, berücksichtigen Gespräch und Zuwendung nicht

und fördern eine mechanistische Fließbandmedizin."

Prof. Dr. med. Lothar Weißbach,

Stiftung Männergesundheit

\section{Bluten für den Quartals-Schluss}

Es gibt ein neues Siegel im bunten Reigen der Krankenhauszertifikate: die Ethik-Klinik (www.ethikklinik.de). Das Konzept ist so naheliegend wie vielversprechend. Ethik-Kliniken sind ausschließlich der weitestgehenden körperlichen Unversehrtheit ihrer Patienten verpflichtet; Indikationen werden hier also nicht nach ökonomischen Zwängen gestellt, sondern nur nach ärztlicher Notwendigkeit und im Einklang mit der Patientenpräferenz. Patienten bekommen so viel ärztliche und pflegerische Zuwendung wie sie wünschen und nur so viel medizinische Versorgung, wie sie brauchen - die aber in höchster Qualität. Damit sind die Ethik-Kliniken die idealen Versorgungseinrichtungen: Für Patienten, weil sie sich darauf verlassen können, dass bei der Wahl der angemessenen Diagnostik und Therapie wirklich nur ihr Wohl ausschlaggebend ist - und nicht etwa Leistungsvereinbarungen, Mindestmengen, Auslastung und der betriebswirtschaftliche Abschluss der Einrichtung. Und für Ärzte, weil sie unter diesen Bedingungen nicht nur ihrem Job, sondern ihrer Berufung nachgehen dürfen: Menschen zu helfen und Leid zu lindern - und das ohne Bevormundung durch die pseudo-akademisierten Drückerkolonnen sogenannter Ökonomen.

Einen Haken hat das Siegel dennoch. Im gesundheitspolitischen Rahmen einer aktionistischen und auf das betriebswirtschaftliche Ergebnis ausgerichteten Medizin kann sich ethisch angemessene Versorgung nicht rechnen. Wer diese bekommen möchte, muss etwas mehr bezahlen als EBM und DRG für die Krankenabfertigung vorsehen. Ethikkliniken bieten diese Behandlung nur Privatversicherten an. Der GKV-Patient bleibt leider draußen. Ähnlich wie beim Bio-Siegel zahlen Betroffene freiwillig dafür, dass sie das bekommen, was ihnen gut tut. Das ist in der heutigen Versorgung häufig weniger als sie in einem Krankenhaus der Regelversorgung bekämen: keine Chemotherapie für asymptomatische, gebrechliche Patienten; Prostatektomie nur dann, wenn eine Überwachung wirklich nicht infrage kommt oder keine schwerwiegenden Begleiterkrankungen vorliegen; keine neue Hüfte für 90jährige durchmetastasierte Patienten.

Das ist ungerecht. Der GKV-Patient kann sich nicht freikaufen. Er muss bluten für die Gesundheitswirtschaft. Damit Mindestmengenvorgaben und Quartalsabschluss stimmen, wird er operiert und anderweitig therapiert, was die Kasse hergibt, egal ob die Indikation aus ärztlicher Sicht gerechtfertigt ist.

Sie meinen, das sei eine schamlose Übertreibung? Naja - die Ethik-Klinik schon. Die ist nicht nur übertrieben, die ist sogar gelogen. Es gibt kein Siegel dafür, dass Patienten aus ethisch-ärztlicher Sicht angemessen behandelt werden. Was aber die Regelversorgung betrifft, so sind die Beispiele alles andere als überzogen. Davon liest man natürlich nicht in den Fachzeitschriften und den Hochglanzbroschüren der Kliniken. Man hört offiziell auch nicht auf Tagungen und Kongressen davon. Aber zum Beispiel abends beim Wein hat fast jeder reflektierte Kollege ähnliche Fälle zu berichten - und erzählt, wie sehr er unter dieser Kommerzialisierung der Medizin leidet. Unserer Fachgesellschaft fehlte der Mut, dieses Thema auf dem Jahreskongress in Düsseldorf zu diskutieren.

Als Arzt ist man leicht versucht, die gesundheitspolitischen Rahmenbedingungen anzuklagen. Die gesetzlichen und die Honorarvorgaben belohnen den Aktionismus, berücksichtigen Gespräch und Zuwendung nicht und fördern eine mechanistische Fließbandmedizin. Das tun auch die Ökonomen (schwache Klinikleiter, Geschäftsführer, Controller), die jetzt im Gesundheitswesen das Sagen haben und die Leistungsvorgaben machen. Mit Bertolt Brecht, der ja schon im vorletzten Editorial so Erhellendes zur Situation der Urologen beitrug, könnte man die Ärzte entschuldigen: „Wir wären gut, anstatt so roh, doch die Verhältnisse, sie sind nicht so."

Allein, das greift zu kurz. Bei allen Widrigkeiten trägt der behandelnde Arzt dennoch die Endverantwortung für den ihm anvertrauten Patienten. Er muss sich bewusst machen, dass jede medizinisch nicht notwendige Maßnahme ein persönliches Schicksal nachhaltig beeinflussen kann. Kasuistiken, die dies belegen gibt es zuhauf. Wer im Sinne des Patientenwohls gegen die Ökonomie entscheidet, braucht im derzeitigen Gesundheitssystem mitunter Heldenmut. Das ist bitter. „Unglücklich das Land, das Helden nötig hat", sagt Brecht. Wie recht er hat!

Ihr

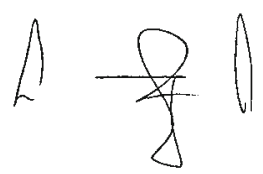

